\title{
Improving the Output Power Stability of a High Concentration Photovoltaic System with Supercapacitors: A Preliminary Evaluation
}

\author{
Yu-Pei Huang and Peng-Fei Tsai \\ Department of Electronic Engineering, National Quemoy University, Kinmen 892, Taiwan \\ Correspondence should be addressed to Yu-Pei Huang; tim@nqu.edu.tw
}

Received 7 September 2014; Accepted 12 December 2014

Academic Editor: Mo Li

Copyright (C) 2015 Y.-P. Huang and P.-F. Tsai. This is an open access article distributed under the Creative Commons Attribution License, which permits unrestricted use, distribution, and reproduction in any medium, provided the original work is properly cited.

\begin{abstract}
The output power of a high concentration photovoltaic (HCPV) system is very sensitive to fluctuating tracking errors and weather patterns. To help compensate this shortcoming, supercapacitors have been successfully incorporated into photovoltaic systems to improve their output power stability. This study examined the output power stability improvement of an HCPV module with a supercapacitor integrated into its circuit. Furthermore, the equivalent model of the experimental circuit is presented and analyzed. Experimental results suggest that integrating a supercapacitor into an HCPV module could improve its output power stability and further extend its acceptance angle. This paper provides preliminary data of the improvement and its evaluation method, which could be utilized for further improvements to an HCPV system.
\end{abstract}

\section{Introduction}

High concentration photovoltaic (HCPV) systems employing high-efficiency III-V solar cells provide higher expected conversion efficiency than conventional photovoltaic systems. Inexpensive optical concentrators are used in HCPV systems to focus the sunlight onto a small area, thereby reducing the required amount of expensive solar cell area. However, most HCPV concentrators use only direct solar radiation; hence, they must integrate an automatic sun tracking system to maintain direct sunlight on the cells $[1,2]$. Among the external disturbances that affect tracking accuracy are fluctuating weather patterns, temperature changes, wind loading, low direct normal irradiation (DNI) conditions, partial shading, and soiling effects. Such tracking errors fluctuate rapidly and introduce uncertainty into the HCPV system's output power $[1,3]$. One method to deal with such output power fluctuations is to use modified maximum power point tracker (MPPT) algorithms; however, the inherent complexity of these methods is the major drawback [4-6]. Another approach uses a model predictive control algorithm for photovoltaic panel orientation with the aim of maximizing the photovoltaic system power production [7]; however, this technique requires local meteorology and geographical data input.

Supercapacitors, also known as electrochemical double layer capacitors (EDLCs) or ultracapacitors, have been developed for many applications in recent years. A power supply system often utilizes supercapacitors to enable them to operate without a primary power source. Supercapacitors do not experience aging effects, irreversible chemical reactions, or dry-up problems; further, they have a higher power density than that of batteries, which is why they provide greater power over a short time period. Despite behaving like common capacitors, supercapacitors have a significantly higher capacitance. Given the above advantages, several designs have incorporated supercapacitors into their photovoltaic systems [8-10]; nevertheless, little has been reported on incorporating supercapacitors into HCPV systems.

The aim of this study was to evaluate the output power stability improvement of an HCPV system with incorporated supercapacitors. We integrated supercapacitors into the HCPV module circuit to compensate for the gap between 
the output from the module and the load. Different capacitances of the supercapacitors were examined under different misalignment angles of the experimental HCPV module. The evaluation result indicated that output power stability could be improved using the proposed HCPV circuit model.

\section{Methodology}

2.1. HCPV Cells Model. III-V multijunction solar cells are mostly employed in HCPV systems, for which the equivalent circuit model of a triple-junction solar cell is presented in Figure 1. A triple-junction solar cell can be considered as being composed of three subcells connected in series. Here, the single-diode model combines the reverse saturation current from recombination in the depletion and the quasineutral regions [11-13]. The cell $I-V$ curve can be mathematically expressed as

$$
\begin{gathered}
V_{\text {Cell }}=\sum_{i=1}^{3} V_{i}, \\
I_{\text {Cell }}=I_{L, i}-I_{0, i}\left\{\exp \left[\frac{q\left(V_{i}+I_{\text {Cell }} R_{S, i}\right)}{n_{i} k T}\right]-1\right\} \\
-\frac{\left(V_{i}+I_{\text {Cell }} R_{S, i}\right)}{R_{H, i}}, \quad i=1,2,3,
\end{gathered}
$$

where $i$ indicates the subcells $(1=$ top, $2=$ middle, and $3=$ bottom), $I_{L}$ is the light generated photocurrent, $I_{0}$ represents the diode reverse saturation current, $q$ is the electrical charge of the electron, $n$ denotes the diode ideality factor, $k$ is Boltzmann's constant, and $T$ is the absolute temperature. Further, $R_{S}$ and $R_{H}$ are the series resistance and shunt resistance, respectively. Assuming the shunt resistance is sufficiently large to be neglected [12], the open-circuit voltage $V_{\mathrm{OC}}$ can be obtained by setting $I_{\text {Cell }}=0$

$$
\begin{gathered}
V_{\mathrm{OC}}=\frac{k T}{q}\left[n_{\mathrm{top}} \ln \left(\frac{I_{L, \text { top }}}{I_{0, \text { top }}}+1\right)+n_{\text {mid }} \ln \left(\frac{I_{L, \text { mid }}}{I_{0, \text { mid }}}+1\right)\right. \\
\left.+n_{\text {bot }} \ln \left(\frac{I_{L, \text { bot }}}{I_{0, \text { bot }}}+1\right)\right],
\end{gathered}
$$

where top, mid, and bot represent the top subcell, middle subcell, and bottom subcell, respectively. Further, $k$ is Boltzmann's constant, $T$ is the absolute temperature, and $q$ is the electrical charge of the electron.

In general, triple-junction solar cells are often monolithic devices which incorporate only two electrical terminals. The short-circuit current $I_{\mathrm{SC}}$ of the triple-junction solar cell is limited by the smaller photocurrent of the top and middle subcells [11]. In addition, the $V_{\mathrm{OC}}$ of the triple-junction solar cell can be obtained from the reference $V_{\text {OC }}$ at one sun by applying a logarithmic correction with concentration using $[14,15]$

$$
V_{\mathrm{OC}}(\mathrm{CR})=V_{\mathrm{OC}}(1)+\frac{n k T}{q} \ln \mathrm{CR}
$$

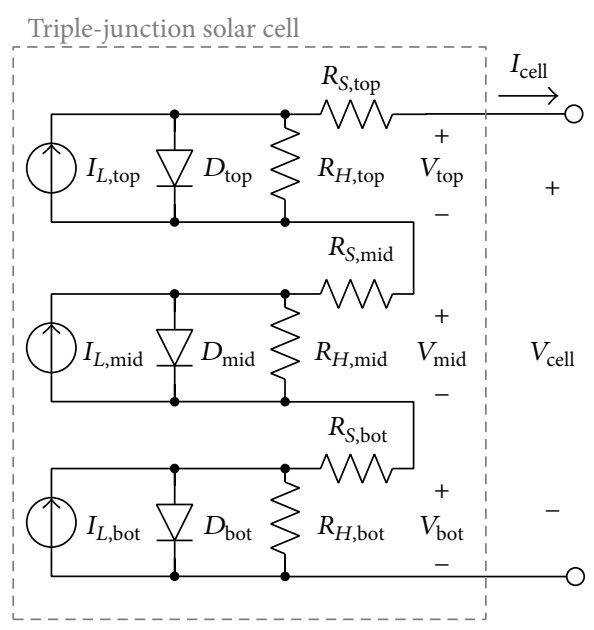

FIGURE 1: Equivalent circuit model for a triple-junction solar cell.

where

$$
\mathrm{CR} \equiv \frac{I_{L}(\mathrm{CR})}{I_{L}(1)}
$$

is the concentration ratio, which can be expressed as the ratio of the photocurrent $I_{L}$ of the triple-junction solar cell at CR-suns $(\mathrm{CR} \times)$ to that at one-sun $(1 \times)$ intensity. Further, $V_{\mathrm{OC}}(\mathrm{CR})$ and $V_{\mathrm{OC}}(1)$ are open-circuit voltages at CR-suns and one-sun intensities, respectively, $n$ is the effective diode ideality factor of the triple-junction solar cell, $k$ is Boltzmann's constant, $T$ is the absolute temperature, and $q$ is the electrical charge of the electron.

The maximum power of an HCPV module can be obtained by

$$
P_{\mathrm{mp}}=\mathrm{FF} \cdot V_{\mathrm{OC}} \cdot I_{\mathrm{SC}}=V_{\mathrm{mp}} \cdot I_{\mathrm{mp}},
$$

where $P_{\mathrm{mp}}$ and FF are the maximum power and fill factor of the module, respectively, while $V_{\mathrm{mp}}$ and $I_{\mathrm{mp}}$ are the operating voltage and current at maximum power point (MPP).

2.2. Supercapacitor Model. Figure 2 illustrates the classical equivalent circuit model for the supercapacitor [16]. The model consists of three components: capacitance, equivalent series resistance (ESR), and equivalent parallel resistance (EPR). As a loss term that models the internal heating in a capacitor, ESR is the dominant factor during charging and discharging. Further, EPR models the current leakage effect and impacts the long-term energy storage performance of a supercapacitor, and $C$ is the capacitance. Equations (6)(8) describe the ESR, EPR, and terminal voltage of a supercapacitor, respectively.

$$
\begin{gathered}
\mathrm{ESR}=\frac{\Delta V}{\Delta i}, \\
\mathrm{EPR}=\frac{-\left(t_{2}-t_{1}\right)}{\ln \left(V_{2} / V_{1}\right) C}, \\
V_{C}=\mathrm{ESR} \cdot i_{C}+\frac{1}{C} \int\left(i_{C}-\frac{e_{C}}{\mathrm{EPR}}\right) d t+V_{C_{-} \text {init }},
\end{gathered}
$$




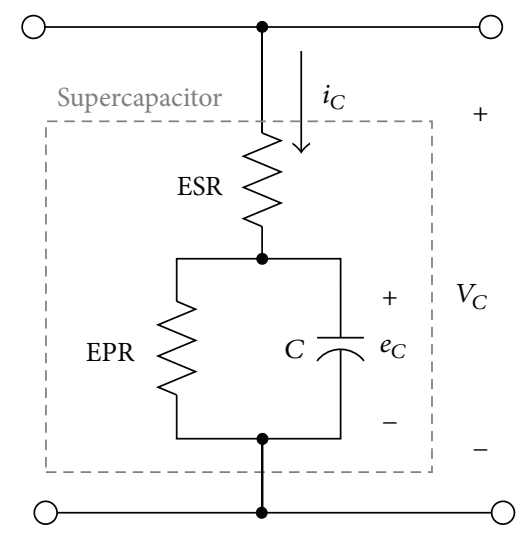

FIgURE 2: Equivalent circuit of a supercapacitor.

where $\Delta V$ and $\Delta i$ denote change in voltage and current at the initiation of load, respectively. Further, $V_{1}$ is the initial self-discharge voltage at $t_{1}, V_{2}$ represents the self-discharge voltage at $t_{2}$, and $C$ refers to the rated capacitance. And, $V_{C}$ represents the terminal voltage of the supercapacitor while $V_{C \text { init }}$ is its initial voltage, $i_{C}$ is the capacitor current, and $e_{C}$ denotes the terminal voltage of EPR and $C$.

2.3. Experimental Circuit. Usually, an HCPV module is composed of a number of triple-junction solar cells connected in series. For simplicity, the experimental module in this study was composed of only one triple-junction solar cell, one bypass diode, and one supercapacitor, the experimental circuit of which is illustrated in Figure 3. Here, a conventional HCPV cell connection, comprising a triple-junction solar cell and a bypass diode, was modified by incorporating a supercapacitor. Tables 1 and 2 list the characteristics of the experimental triple-junction solar cell (T1000, Emcore, USA) and supercapacitors (MPL, PAC Electronics, Taiwan), respectively.

2.4. Charging and Discharging of Supercapacitors. Integrating a supercapacitor into a solar cell circuit allows it to operate without a primary power source by compensating dynamic gaps between the output from the solar cell and the load due to charging and discharging operations. The residual energy of a supercapacitor is given by

$$
E_{\mathrm{CAP}}=\frac{1}{2} C V_{C}^{2}
$$

where $E_{\mathrm{CAP}}$ is the energy stored in the supercapacitor, $C$ is the capacitance, and $V_{C}$ is the terminal voltage of the supercapacitor. $E_{\mathrm{CAP}}$ increases or decreases dynamically during charging and discharging operations, and when charging, current flows from the solar cell to the supercapacitor. In this period, the supercapacitor voltage $V_{C}$ and charging current $i_{C}$ can be calculated by (8), as described in the previous section. On the other hand, during discharging operation, current flows from the supercapacitor to the load. The discharging
TABLE 1: Characteristics of the experimental triple-junction solar cell.

\begin{tabular}{lc}
\hline Characteristics & Spec \\
\hline$V_{\mathrm{OC}}$ & $3.05[\mathrm{~V}]$ \\
$I_{\mathrm{SC}}$ & $1.96[\mathrm{~A}]$ \\
$V_{\mathrm{mp}}$ & $2.70[\mathrm{~V}]$ \\
$I_{\mathrm{mp}}$ & $1.90[\mathrm{~A}]$ \\
$P_{\mathrm{mp}}$ & $5.13[\mathrm{~W}]$ \\
Efficiency & $36.23 \%$ \\
Area & $0.3025\left[\mathrm{~cm}^{2}\right]$ \\
\hline
\end{tabular}

Tested under $850 \mathrm{~W} / \mathrm{m}^{2}, 25^{\circ} \mathrm{C}, 485 \times \mathrm{CR}$ with Fresnel lens optics.

TABLE 2: Characteristics of the experimental supercapacitors.

\begin{tabular}{lcc}
\hline Rated cap. $[\mathrm{F}]$ & ESR $[\Omega]$ & Stored energy $[\mathrm{Wh}]$ \\
\hline 0.5 & 1.40 & 0.002 \\
1.0 & 0.76 & 0.004 \\
2.0 & 0.41 & 0.008 \\
3.0 & 0.26 & 0.012 \\
4.0 & 0.20 & 0.016 \\
5.0 & 0.15 & 0.020 \\
\hline
\end{tabular}

Measured at $25^{\circ} \mathrm{C}$, rate voltage $=5.4 \mathrm{~V}$.

circuit can be modeled as a simple $R C$ network, for which the voltage decay after $\Delta t$ time can be expressed as

$$
V_{C}(t+\Delta t)=V_{C}(t) \cdot e^{-\Delta t / R C},
$$

where $V_{C}$ is the terminal voltage of the supercapacitor, $C$ is the capacitance, and $R$ denotes the resistance of the load.

The charging efficiency of the supercapacitor in the experimental circuit is determined by solar irradiance, temperature, the MPP voltage of the triple-junction solar cell $V_{\mathrm{mp}}$, and the supercapacitor voltage $V_{C}$. In addition, the charging efficiency is strongly related to the mismatch between $V_{\mathrm{mp}}$ and $V_{C}$ [17]. In this study, the triple-junction solar cell and the supercapacitor were directly connected in parallel, and $V_{C}$ was controlled to approximately equal $V_{\mathrm{mp}}$ by appropriately adjusting the load resistance.

2.5. Experiment Setup. The output power of an HCPV module decreases rapidly when the tracking error exceeds the acceptance angle [3]. In the HCPV field, the acceptance angle is defined as the misalignment angle of the sunlight at which point a module still delivers at least $90 \%$ of its maximum power. The acceptance angle of the experimental module is around $0.5^{\circ}$. To evaluate the output power stability improvement of the proposed experimental circuit, a sun tracking system adjusted the misalignment angle of the sunlight. Figure 4 demonstrates that when the misalignment angle is larger than the acceptance angle, the focus shifts from the area of the triple-junction solar cell, which would likely cause the output power of the experimental module to decrease rapidly. To simulate output power fluctuations of the solar cell caused by tracking errors, the misalignment angle of the sunlight was varied from zero degrees to larger than the acceptance angle in this study. 


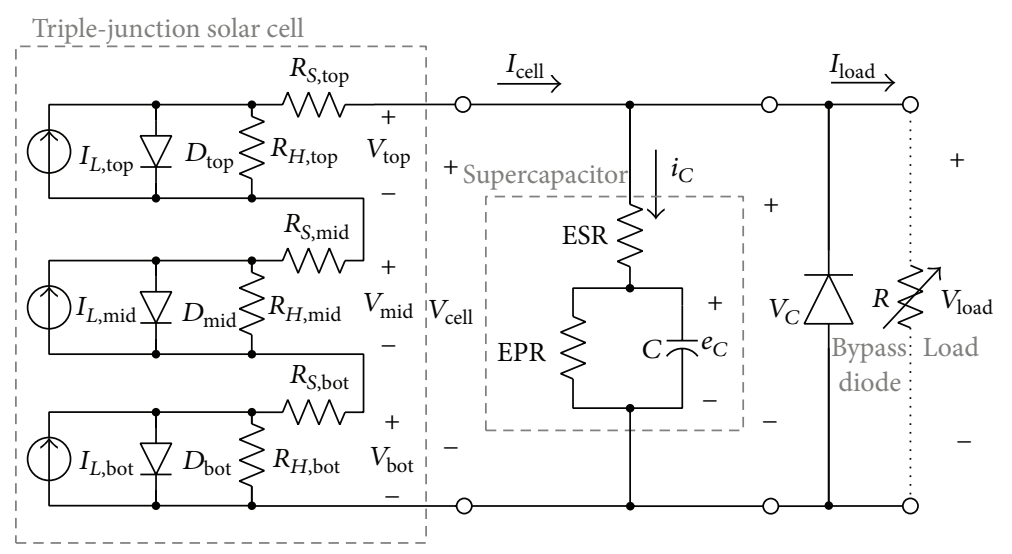

FIGURE 3: Experimental circuit diagram.
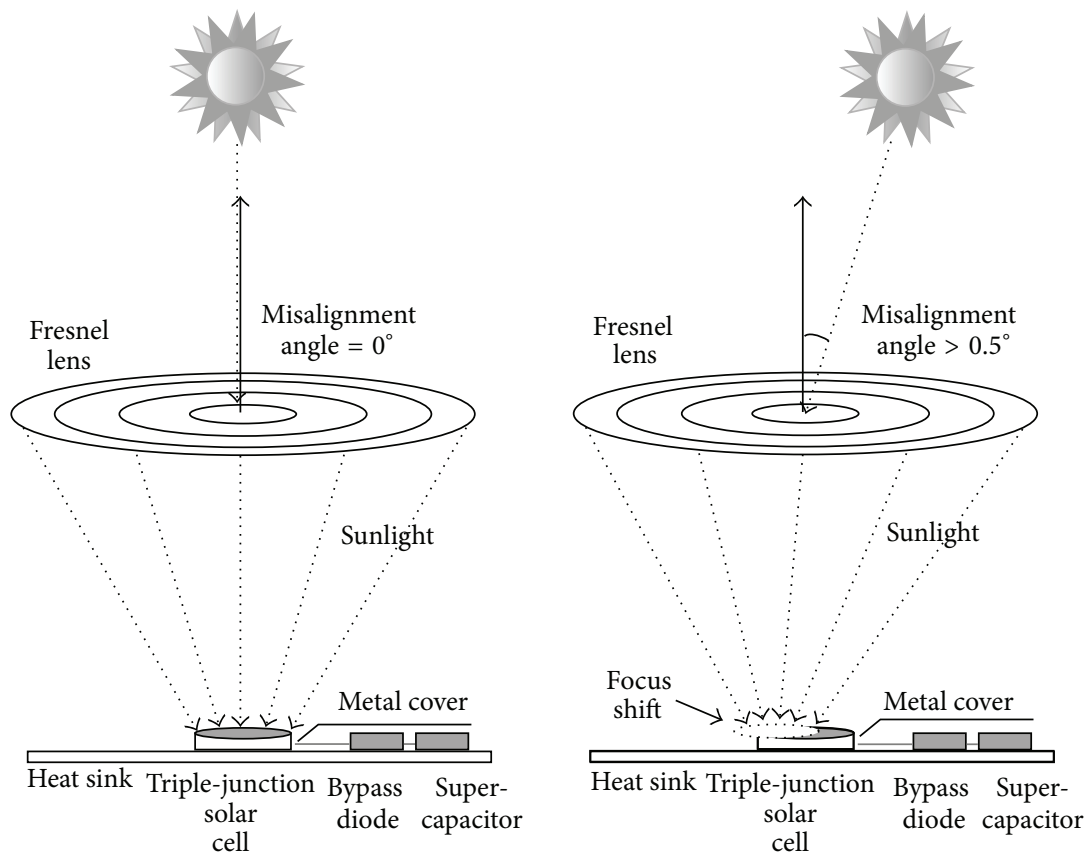

FIGURE 4: Focus shifts of the experimental module.

To evaluate the power stability improvement of the experimental circuit, a comprehensive sun tracker (GST-200, Green Source Technology, Taiwan), which includes a sun sensor, actuators, frame, and load, was installed at the National Quemoy University test site in Kinmen County, Taiwan. The block diagram of the experimental system is illustrated in Figure 5. As can be seen, the experimental HCPV module was mounted onto the frame, the output voltage of which was measured by a differential amplifier. The output of the amplifier was connected to a data acquisition (DAQ) module with 16-bit resolution ADCs (DAQPad-6015, National Instrument, USA) and then transmitted to a PC for analysis. A diagnostic instrument to measure the misalignment angle of the sunlight (Trac-Stat SL1, Pract Engineering, USA) with a typical relative accuracy of $0.02^{\circ}$ and a resolution of $0.01^{\circ}$ was also mounted onto this system. The analog outputs of the Trac-Stat SL1 were sampled by the ADCs of the DAQ module, after which DNI levels were recorded by using a calibrated pyrheliometer (NIP, The Eppley Laboratory, USA). The analog outputs of the pyrheliometer were connected to a second-order Butterworth low-pass filter (cut-off frequency $10 \mathrm{~Hz}$ ) for noise elimination. The output of the filter was then converted by the ADCs of the DAQ module into digital format and transmitted to the PC. Ambient temperature/humidity were sensed and recorded by a thermal/humidity meter (TES-1365, TES Electrical Electronic Corporations, Taiwan). The azimuth and altitude rotation angles of the tracker frame were controlled by the tracking control system via the motors and actuators; consequently, the misalignment angle of the sunlight to the HCPV module could be controlled by the PC.

Two separate experiments were conducted to examine the effects of capacitance value and maximum misalignment 


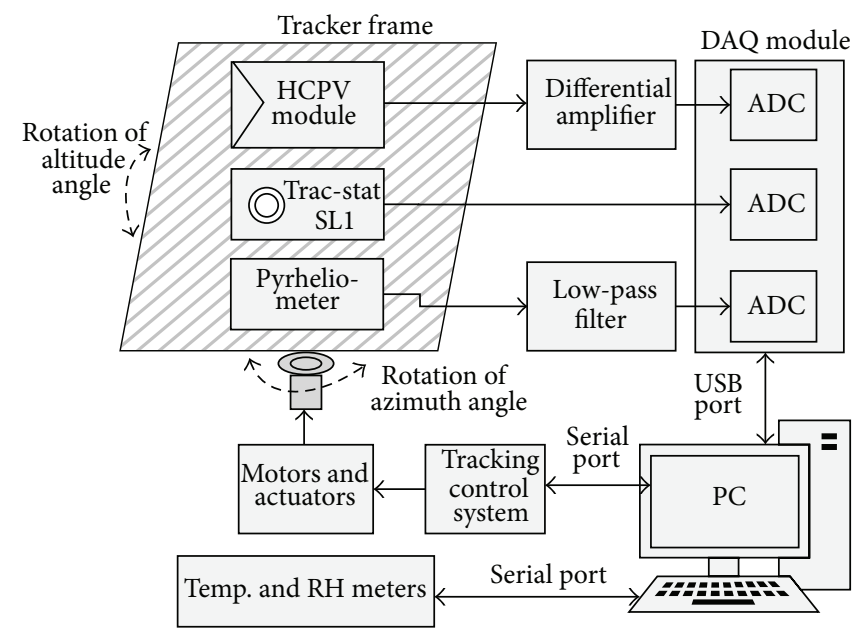

FIGURE 5: Block diagram of the experimental system.

angle of the module on the output power decay of the HCPV module. In Experiment 1, capacitance of the connected supercapacitor $C$ was the independent variable. During this experiment, the maximum misalignment angle $\theta_{\text {mis,max }}$ was controlled at $1^{\circ}$, which is larger than the acceptance angle of $0.5^{\circ}$. In this capacitance experiment, the range of $C$ extended from 0.5 to $7 \mathrm{~F}$. Due to the limited selections of the supercapacitors, the capacitance of the experimental supercapacitors was selected as $0.5,1,3,5$, and 7 F. Experiment 2 used the maximum misalignment angle $\theta_{\text {mis,max }}$ as the independent variable, while $C$ was fixed at $7 \mathrm{~F}$. In this second experiment, the $\theta_{\text {mis,max }}$ was regulated over the range of $1^{\circ}$ to $3^{\circ}$. During the regulation process of Experiment 1 and Experiment 2, the misalignment angle $\theta_{\text {mis }}$ was varied from $0^{\circ}$ to $\theta_{\text {mis,max }}$ by rotating the altitude angle of the tracker with constant angular velocity of $\theta_{\text {mis,max }}$ degrees per 10 seconds. In addition, the voltage of the load $V_{\text {load }}$ was continually logged for output power $P_{\text {load }}$ calculation, which is given by $P_{\text {load }}=V_{\text {load }}^{2} / R$. The operating point of the triple-junction solar cell was mainly determined by the irradiance and the load resistance. For simplicity, experiments were conducted under sunny and low wind velocity conditions. In addition, the resistance of the load $R$ was adjusted via a variable resistor to approximately $10 \Omega$, which corresponds to a load current of around 250-300 $\mathrm{mA}$. As such, the operating voltage of the triple-junction solar cell was set over the range of $V_{\mathrm{OC}}$ to $V_{\mathrm{mp}}$.

\section{Experimental Results}

The main purpose of this study was to evaluate the output power stability improvement of an HCPV module with supercapacitors. According to the evaluation results of the experimental module, it is evident that adding a supercapacitor to the HCPV cell circuit improves the stability of its output power. As illustrated by Figure 6, when the misalignment angle $\theta_{\text {mis }}$ was regulated from $0^{\circ}$ to $1^{\circ}$ and then back from $1^{\circ}$ to $0^{\circ}$, the output power of the experimental module $P_{\text {load }}$ with higher supercapacitor capacitance decayed less during the regulation process. The data of the minimum voltage and

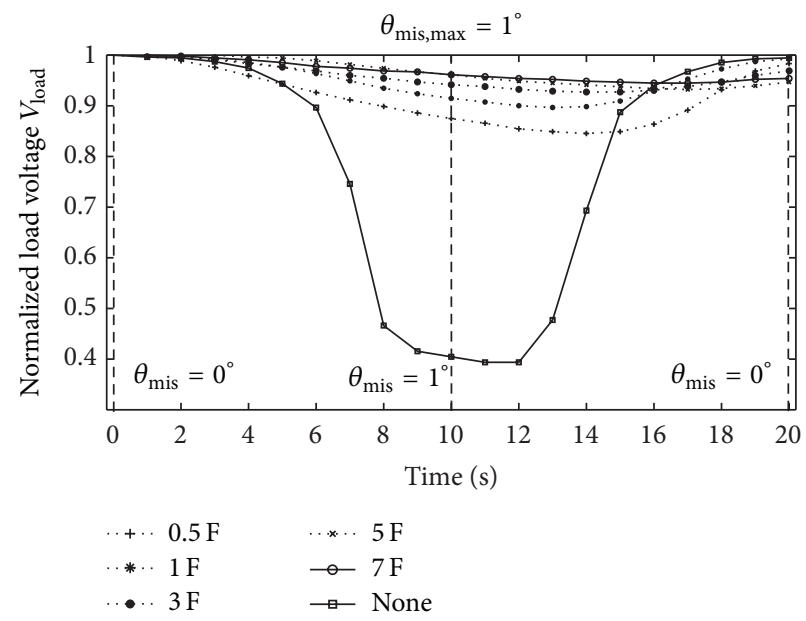

FIGURE 6: Output power stability with different capacitance of supercapacitors.

TABLE 3: Minimum voltage and power of the load resistor under a $1^{\circ}$ misalignment angle with different supercapacitor capacitances.

\begin{tabular}{lcc}
\hline$C[\mathrm{~F}]$ & $V_{\text {load }} \operatorname{min.}[\%]$ & $P_{\text {load }} \operatorname{min.~[\% ]~}$ \\
\hline None & 39.38 & 15.51 \\
0.5 & 84.53 & 71.45 \\
1.0 & 89.67 & 80.41 \\
3.0 & 92.74 & 86.01 \\
5.0 & 93.23 & 86.92 \\
7.0 & 94.77 & 89.81 \\
\hline
\end{tabular}

Tested under $27^{\circ} \mathrm{C}$ ambient temperature, DNI $=623 \mathrm{~W} / \mathrm{m}^{2}$.

minimum power with different supercapacitor capacitance is addressed in Table 3. During the regulation process with a $1^{\circ}$ misalignment angle and $7 \mathrm{~F}$ supercapacitor, $V_{\text {load }}$ remained above $94.77 \%$ of its original value, which corresponded to around $90 \%(89.81 \%)$ of the output power $P_{\text {load }}$. On the other 


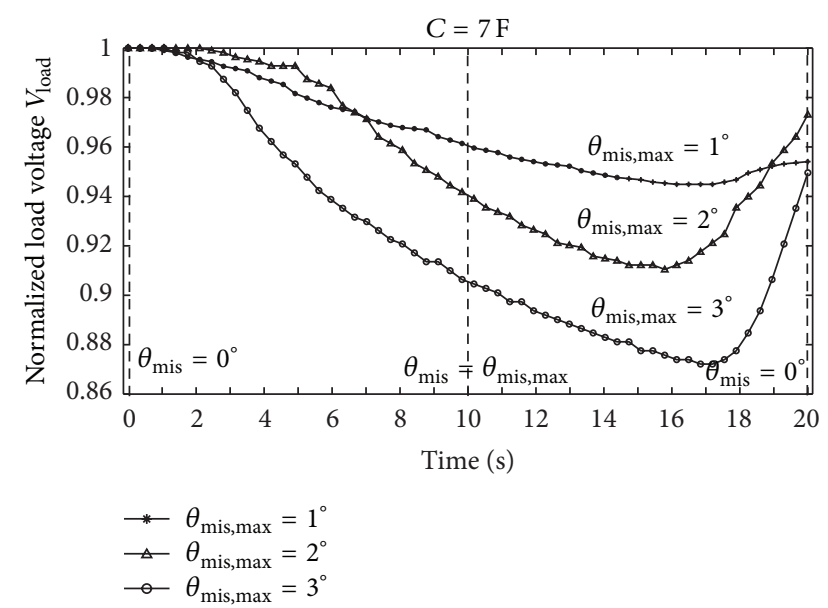

FIGURE 7: Output power stability with $7 \mathrm{~F}$ supercapacitor under different misalignment angles.

hand, $V_{\text {load }}$ of the experimental module without a supercapacitor dropped dramatically to approximately $39.38 \%$ of its original value, which corresponded to around only $15.51 \%$ output power $P_{\text {load }}$. It appears that the acceptance angle of the experimental module could be extended from $0.5^{\circ}$ to around $1^{\circ}$ under the experimental conditions by adding a $7 \mathrm{~F}$ supercapacitor into the circuit.

To further examine the output power stability of the experimental module with a $7 \mathrm{~F}$ supercapacitor under different misalignment angles, the maximum misalignment angles $\theta_{\text {mis,max }}$ were set as $1^{\circ}, 2^{\circ}$, and $3^{\circ}$ for misalignment angle regulation, respectively. As illustrated in Figure $7, V_{\text {load }}$ was more stable under the smallest misalignment angle during the regulation process. Furthermore, it can be seen in both Figures 6 and 7 that the $V_{\text {load }}$ decay speed caused by the misalignment of sunlight and recover speed might, respectively, be slowed down by the supercapacitor discharging and charging effects.

\section{Discussion and Conclusion}

The improvement of output power stability of an HCPV module with a supercapacitor was evaluated in this study. According to the results of the experimental module under a $1^{\circ}$ temporary misalignment angle of sunlight, the voltage of the $10 \Omega$ load resistor $V_{\text {load }}$ remained above $94.77 \%$ of its original value, which corresponded to around $90 \%$ of output power. Therefore, it is evident that the output power stability and acceptance angle of an HCPV module might be improved by adding supercapacitors into the circuit. It also appears that the acceptance angle of the experimental module was extended from $0.5^{\circ}$ to around $1^{\circ}$ under the experimental conditions with a $7 \mathrm{~F}$ supercapacitor. Furthermore, with fixed capacitance supercapacitor added, $V_{\text {load }}$ appeared more stable under smaller temporary misalignment angles of sunlight. To our knowledge, this is the first study to investigate the output power stability improvement of HCPV modules with embedded supercapacitors. The preliminary results suggest that this approach appears to be effective in improving an HCPV module's output power stability and acceptance angle. Our study provides exploratory data of the improvement and the evaluation method used, which could be employed for further improving HCPV systems. However, some limitations are worth noting: this study examined a single triple-junction solar cell with one supercapacitor; as such, future studies could explore integrating supercapacitors to a conventional HCPV module with a number of solar cells connected in series and parallel. In addition, the load resistor could be replaced by an active load for evaluations under different environmental conditions. Further, this study examined the output power stability in the static state of the HCPV modules. Future studies should examine the thermal effect of the cell under long-term dynamic conditions.

\section{Conflict of Interests}

The authors declare that there is no conflict of interests regarding the publication of this paper.

\section{Acknowledgment}

The authors would like to thank the National Science Council of Taiwan, for financially supporting this research under contract no. MOST103-2221-E-507-004.

\section{References}

[1] A. L. López and V. M. Andreev, Concentrator Photovoltaics, Springer, 2010.

[2] L. M. Fraas and L. D. Partain, Solar Cells and Their Applications, John Wiley \& Sons, New York, NY, USA, 2010.

[3] Y.-P. Huang, K.-W. Weng, and P.-F. Tsai, "Evaluation of the stability improvement in power generation of a concentration photovoltaic module with supercapacitors," in Proceedings of the 38th IEEE Photovoltaic Specialists Conference (PVSC '12), pp. 941-943, June 2012.

[4] K. Ishaque and Z. Salam, "A review of maximum power point tracking techniques of PV system for uniform insolation and partial shading condition," Renewable and Sustainable Energy Reviews, vol. 19, pp. 475-488, 2013.

[5] X. Gao, S. Li, and R. Gong, "Maximum power point tracking control strategies with variable weather parameters for photovoltaic generation systems," Solar Energy, vol. 93, pp. 357-367, 2013.

[6] T. L. Nguyen and K.-S. Low, "A global maximum power point tracking scheme employing DIRECT search algorithm for photovoltaic systems," IEEE Transactions on Industrial Electronics, vol. 57, no. 10, pp. 3456-3467, 2010.

[7] M. Gulin, M. Vašak, and N. Perić, "Dynamical optimal positioning of a photovoltaic panel in all weather conditions," Applied Energy, vol. 108, pp. 429-438, 2013.

[8] P. Thounthong, V. Chunkag, P. Sethakul, S. Sikkabut, S. Pierfederici, and B. Davat, "Energy management of fuel cell/solar cell/supercapacitor hybrid power source," Journal of Power Sources, vol. 196, no. 1, pp. 313-324, 2011.

[9] M. E. Glavin, P. K. W. Chan, S. Armstrong, and W. G. Hurley, "A stand-alone photovoltaic supercapacitor battery hybrid energy storage system," in Proceedings of the 13th International Power 
Electronics and Motion Control Conference (EPE-PEMC '08), pp. 1688-1695, September 2008.

[10] M.-E. Choi, S.-W. Kim, and S.-W. Seo, "Energy management optimization in a battery/supercapacitor hybrid energy storage system," IEEE Transactions on Smart Grid, vol. 3, no. 1, pp. 463472, 2012.

[11] P. Rodrigo, E. F. Fernández, F. Almonacid, and P. J. PérezHigueras, "Models for the electrical characterization of high concentration photovoltaic cells and modules: a review," Renewable and Sustainable Energy Reviews, vol. 26, pp. 752-760, 2013.

[12] G. Segev, G. Mittelman, and A. Kribus, "Equivalent circuit models for triple-junction concentrator solar cells," Solar Energy Materials and Solar Cells, vol. 98, pp. 57-65, 2012.

[13] H. Yu-Pei, "A rapid maximum power measurement system for high-concentration photovoltaic modules using the fractional open-circuit voltage technique and controllable electronic load," IEEE Journal of Photovoltaics, vol. 4, no. 6, pp. 1610-1617, 2014.

[14] G. S. Kinsey, P. Hebert, K. E. Barbour, D. D. Krut, H. L. Cotal, and R. A. Sherif, "Concentrator multifunction solar cell characteristics under variable intensity and temperature," Progress in Photovoltaics: Research and Applications, vol. 16, no. 6, pp. 503-508, 2008.

[15] C. Algora and I. Rey-Stolle, "The interest and potential of ultrahigh concentration," in Next Generation of Photovoltaics, A. B. C. López, A. M. Vega, and A. L. López, Eds., vol. 165, pp. 23-60, Springer, Berlin, Germany, 2012.

[16] R. L. Spyker and R. M. Nelms, "Classical equivalent circuit parameters for a double-layer capacitor," IEEE Transactions on Aerospace and Electronic Systems, vol. 36, no. 3, pp. 829-836, 2000.

[17] Z. Liu, X. Y. Yang, S. S. Yang, and J. McCann, "Efficiencyaware: maximizing energy utilization for sensor nodes using photovoltaic-supercapacitor energy systems," International Journal of Distributed Sensor Networks, vol. 2013, Article ID 627963, 11 pages, 2013. 


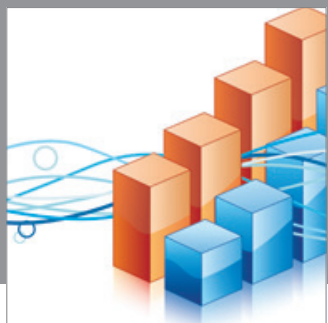

Advances in

Operations Research

mansans

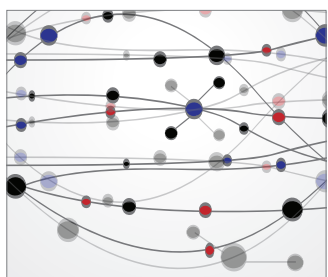

The Scientific World Journal
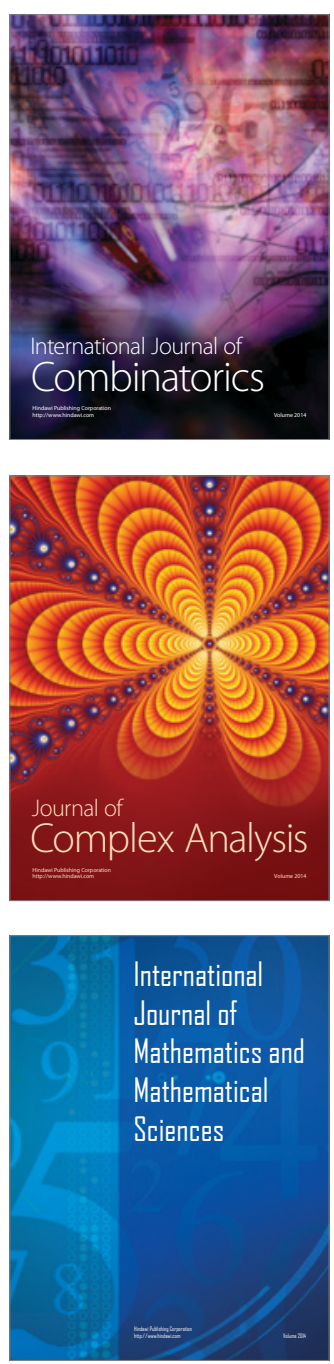
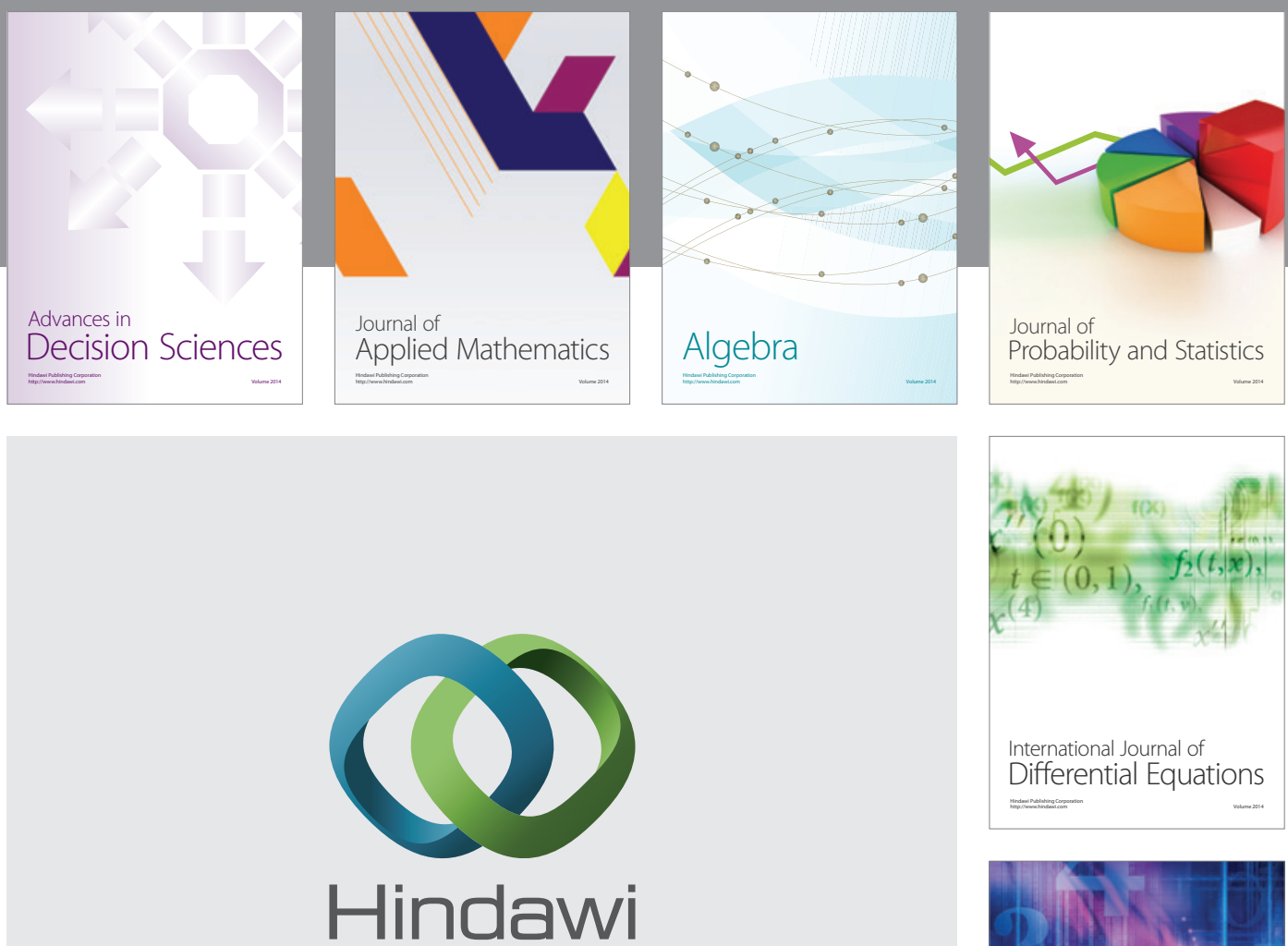

Submit your manuscripts at http://www.hindawi.com
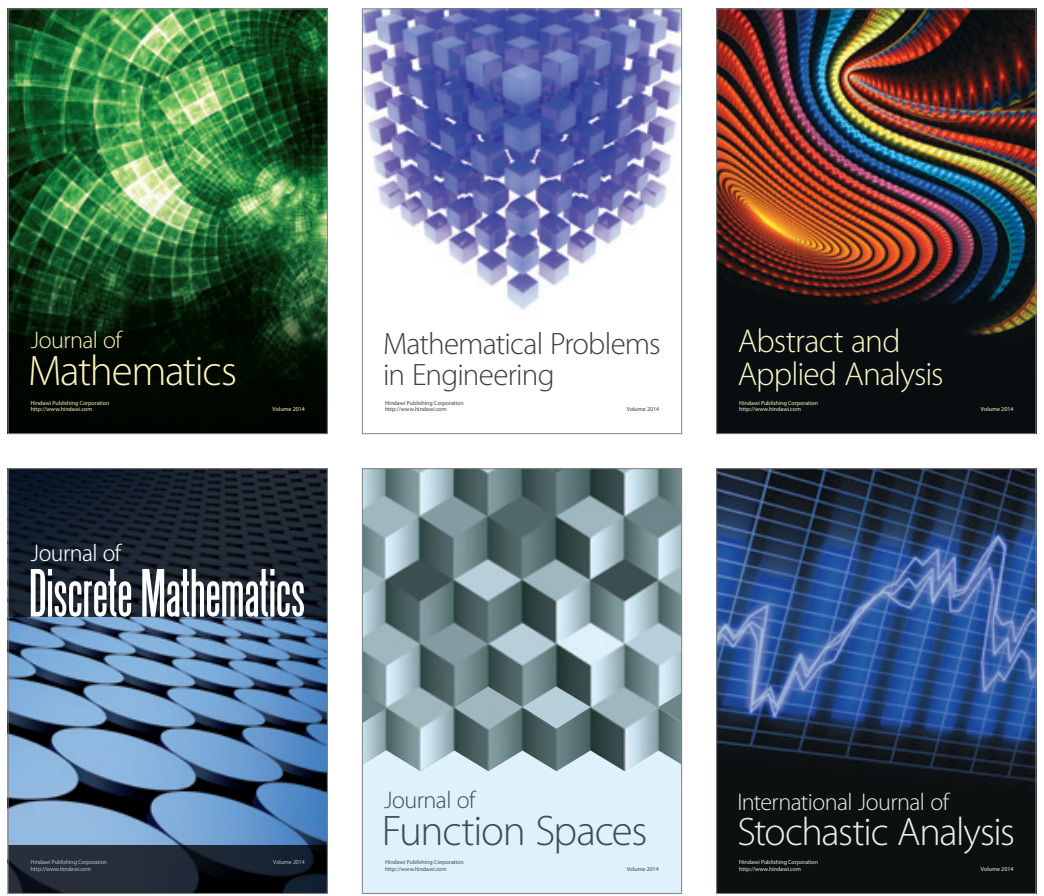

Journal of

Function Spaces

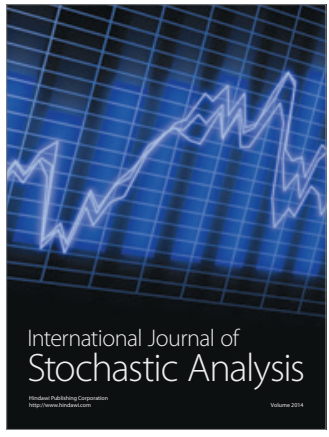

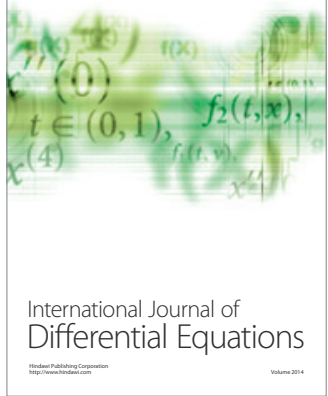
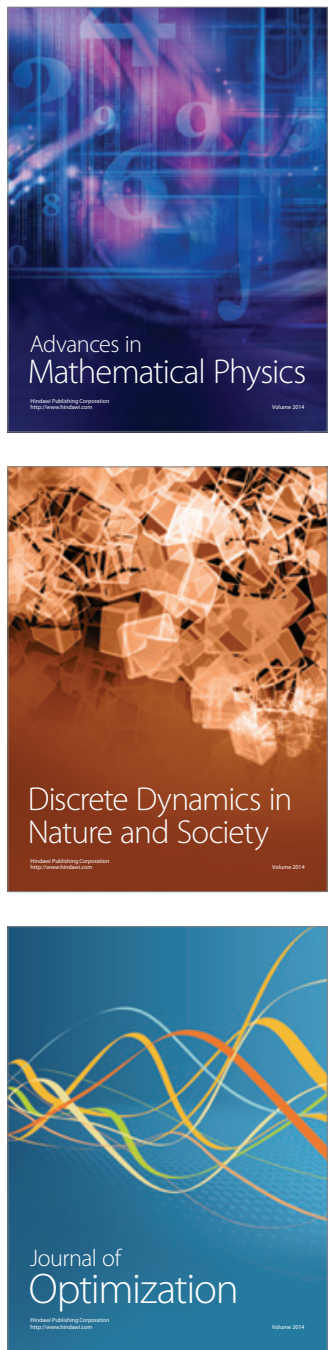